\title{
Discharge Transition Experienced by Korean Older Adults After Hip Fracture Surgery: A Qualitative Study
}

\author{
YoungJi Ko ( $\nabla$ yjko@dhu.ac.kr) \\ Daegu Haany University \\ JuHee Lee \\ Yonsei University \\ Seung-Hoon Baek \\ Kyungpook National University Hospital
}

\section{Research Article}

Keywords: hip fracture, qualitative research, nursing, frail elderly, patient discharge, phase transition

Posted Date: December 22nd, 2020

DOI: https://doi.org/10.21203/rs.3.rs-130312/v1

License: @ (i) This work is licensed under a Creative Commons Attribution 4.0 International License. Read Full License 


\section{Abstract}

Background: This study aimed to explore Korean older adults' experience with discharge transition after hip fracture surgery.

Methods: This was a descriptive qualitative study. Face-to-face interviews following hip fracture surgery were conducted with 12 participants. Data were collected one to two days before discharge and again four weeks after discharge following hip fracture surgery, and were analyzed using qualitative content analysis.

Results: Four main themes were identified: (1) Challenge of discharge transition: unprepared discharge, transfer into other care settings, and eagerness for recovery; (2) Physical and psychological distress against recovery: frail physical state and psychological difficulties; (3) Dependent compliance: absolute trust in healthcare providers, indispensable support from the family, and passive participation in care; and (4) Walking for things they took for granted: hope of walking and poor walking ability.

Conclusions: After the hip fracture surgery, older adults hoped to be able to walk to perform the simple daily chores they earlier took for granted. Considering the physical and psychological frailty of older adults, systematic nursing interventions, including collaboration and coordination with other healthcare professional and settings, are necessary during discharge transition after hip fracture surgery. Invitation for care participation by older adults as well as family involvement should be considered at discharge transitional care in South Korea.

\section{Introduction}

The incidence of hip fractures is increasing with the rapid growth in the older population (1). Fragility fracture of the hip is associated with higher morbidity, mortality, and socioeconomic burden (2). Surgery is a standard treatment (3); however, short hospitalization periods increase patients' concerns as well as the risk for post-surgical complications $(4,5)$. Most older adults are transferred to other care settings due to difficulties in walking or performing activities of daily living (ADL) (6-9). Transitions between care settings are burdensome (10), and older adults with various health problems are particularly vulnerable because they receive limited continuous care (11). Even one year after surgery, many older adults fail to recover their preoperative performance levels on ADL (12). Consequently, the concept of "discharge transition" requires elucidation.

\section{Background}

Discharge is defined as a transition from one state or place to another (13). In a previous study (14), 45.8\% of the discharged older adults reported experiencing significant problems with managing their health care, including the performance of tasks associated with their health and adherence to ongoing care plans. The major transitional difficulties that older adults face after hip fracture are physical function limitations, pain and its management (9). Physical performance, including walking and ADL, is an essential prerequisite for older adults dwelling in a community environment (15). Therefore, it is important to manage pain and attain the best possible ADL performance.

Previous studies on physical recovery after hip fracture have focused mainly on identifying factors affecting it $(12,16)$. Meanwhile, qualitative approaches are appropriate to fully understand the recovery process from the perspective of older adults, and as a fundamental data for nursing interventions to improve the recovery of older adults at the time of discharge $(17,18)$. Some previous qualitative studies have described the recovery experiences of older adults after hip fracture surgery $(4,8,18-20)$.

To the best of our knowledge, there have been few studies describing older adults' experience of discharge transition after hip fracture surgery in South Korea. In South Korea, surgical hospitals provide postoperative care and rehabilitation during hospitalization (21), while in the Western countries, patients are systematically transferred to acute or subacute care facilities after discharge from surgical hospitals (22). These differences might affect the older adults' perceptions and experiences of discharge transition. The recovery of hip fracture surgery requires a long period of time. As older adults struggle to cope with their physical impairments (19), most enter other non-medical facilities or homes after discharge from the hospital where the surgery is performed (21).

In addition to the differences in the health care systems, the recovery experiences of older adults with hip fracture surgery in Asia will differ from those in the Western countries, given that in the family-oriented Asian cultures, the family has the most influence on the patient's life $(23,24)$. Owing to the rapid growth of the older population, the annual incidence of hip fractures in Korea is approximately 82,550 cases, and older adults accounted for $64,366(78 \%)$ of these cases (25). Therefore, there is an urgent need to 
explore the experiences of older adults and their primary caregivers after hip fracture surgery to develop transitional strategies to reduce readmission, morbidity, mortality, and socioeconomic burden (26). The purpose of this study was to describe older adults' experiences with discharge transition after hip fracture surgery in South Korea.

\section{Method}

\section{Design}

This was a descriptive qualitative study based on open-ended and semi-structured interviews.

\section{Participants}

The participants comprised 12 older adults aged 65 years or above who did not have any serious neurological disease, such as stroke or dementia with paralysis and/or cognitive deficits. Only those older adults who understood the study purpose and agreed to participate were recruited in the study. Using convenience sampling, the participants were recruited from a teaching university hospital in South Korea (Table 1). 
Table 1

Demographics of older patients and primary caregivers

\begin{tabular}{|c|c|c|c|c|c|c|c|}
\hline & $\begin{array}{l}\text { Age } \\
\text { (years) }\end{array}$ & $\begin{array}{l}\text { Walking status before hip } \\
\text { surgery }\end{array}$ & $\begin{array}{l}\text { No. of } \\
\text { comorbidities }\end{array}$ & $\begin{array}{l}\text { Type of hip } \\
\text { surgery }\end{array}$ & $\begin{array}{l}\text { Residence } \\
\text { after } \\
\text { discharge }\end{array}$ & $\begin{array}{l}\text { Interviewed } \\
\text { after } \\
\text { discharge }\end{array}$ & $\begin{array}{l}\text { Walking } \\
\text { status after } \\
\text { discharge }\end{array}$ \\
\hline 001 & 78 & $\begin{array}{l}\text { Independent walking in } \\
\text { and outdoors }\end{array}$ & 2 & Internal fixation & $\begin{array}{l}\text { Rehabilitation } \\
\text { hospital }\end{array}$ & Yes & $\begin{array}{l}\text { Immobile } \\
\text { status in } \\
\text { and } \\
\text { outdoors }\end{array}$ \\
\hline 002 & 65 & $\begin{array}{l}\text { Independent walking in } \\
\text { and outdoors }\end{array}$ & 0 & $\begin{array}{l}\text { Total hip } \\
\text { replacement }\end{array}$ & Home & Yes & $\begin{array}{l}\text { Independent } \\
\text { walking } \\
\text { indoors; } \\
\text { using a } \\
\text { walking aid } \\
\text { outdoors }\end{array}$ \\
\hline 003 & 87 & $\begin{array}{l}\text { Independent walking } \\
\text { indoors; using a walking } \\
\text { aid outdoors }\end{array}$ & 5 & Internal fixation & $\begin{array}{l}\text { Long term } \\
\text { care hospital }\end{array}$ & No & $\begin{array}{l}\text { Immobile } \\
\text { status in } \\
\text { and } \\
\text { outdoors }\end{array}$ \\
\hline 004 & 75 & $\begin{array}{l}\text { Independent walking } \\
\text { indoors; using a walking } \\
\text { aid outdoors }\end{array}$ & 6 & Internal fixation & $\begin{array}{l}\text { Long term } \\
\text { care hospital }\end{array}$ & Yes & $\begin{array}{l}\text { Immobile } \\
\text { status in } \\
\text { and } \\
\text { outdoors }\end{array}$ \\
\hline 005 & 80 & $\begin{array}{l}\text { Independent walking in } \\
\text { and outdoors }\end{array}$ & 4 & Internal fixation & $\begin{array}{l}\text { Rehabilitation } \\
\text { hospital }\end{array}$ & Yes & $\begin{array}{l}\text { Using a } \\
\text { walking aid } \\
\text { indoors }\end{array}$ \\
\hline 006 & 65 & $\begin{array}{l}\text { Independent walking } \\
\text { indoors; using a walking } \\
\text { aid outdoors }\end{array}$ & 3 & $\begin{array}{l}\text { Total hip } \\
\text { replacement }\end{array}$ & $\begin{array}{l}\text { Rehabilitation } \\
\text { hospital }\end{array}$ & Yes & $\begin{array}{l}\text { Immobile } \\
\text { status in } \\
\text { and } \\
\text { outdoors }\end{array}$ \\
\hline 007 & 73 & $\begin{array}{l}\text { Independent walking in } \\
\text { and outdoors }\end{array}$ & 2 & Hemiarthroplasty & $\begin{array}{l}\text { Rehabilitation } \\
\text { hospital }\end{array}$ & No & $\begin{array}{l}\text { Immobile } \\
\text { status in } \\
\text { and } \\
\text { outdoors }\end{array}$ \\
\hline 008 & 85 & $\begin{array}{l}\text { Independent walking in } \\
\text { and outdoors }\end{array}$ & 5 & Internal fixation & $\begin{array}{l}\text { Long term } \\
\text { care hospital }\end{array}$ & Yes & $\begin{array}{l}\text { Immobile } \\
\text { status in } \\
\text { and } \\
\text { outdoors }\end{array}$ \\
\hline 009 & 78 & $\begin{array}{l}\text { Independent walking in } \\
\text { and outdoors }\end{array}$ & 2 & $\begin{array}{l}\text { Total hip } \\
\text { replacement }\end{array}$ & $\begin{array}{l}\text { Long term } \\
\text { care hospital }\end{array}$ & Yes & $\begin{array}{l}\text { Immobile } \\
\text { status in } \\
\text { and } \\
\text { outdoors }\end{array}$ \\
\hline 010 & 74 & $\begin{array}{l}\text { Independent walking in } \\
\text { and outdoors }\end{array}$ & 2 & $\begin{array}{l}\text { Total hip } \\
\text { replacement }\end{array}$ & $\begin{array}{l}\text { Long term } \\
\text { care hospital }\end{array}$ & Yes & $\begin{array}{l}\text { Using a } \\
\text { walking aid } \\
\text { outdoors }\end{array}$ \\
\hline 011 & 81 & $\begin{array}{l}\text { Using a walking aid in and } \\
\text { outdoors }\end{array}$ & 4 & Internal fixation & $\begin{array}{l}\text { Long term } \\
\text { care hospital }\end{array}$ & No & Death \\
\hline 012 & 77 & $\begin{array}{l}\text { Using a walking aid in and } \\
\text { outdoors }\end{array}$ & 2 & Internal fixation & Home & Yes & $\begin{array}{l}\text { Using a } \\
\text { walking aid } \\
\text { in and } \\
\text { outdoors }\end{array}$ \\
\hline
\end{tabular}

\section{Data Collection}

From November 2017 to February 2018, data were collected one to two days before discharge and again four weeks after discharge. Of the 12 participants, 3 could not participate in the follow-up interview. Thus, 12 participants were included in the pre-discharge survey and 9 in the post-discharge survey. Pre-discharge interviews were conducted face-to-face in hospital wards. Four weeks after discharge, each older adult was individually interviewed in an outpatient meeting room. Data collection was carefully conducted using 
an interview guide with open-ended and semi-structured questions. Each interview began with the general question, "Tell me about your discharge experience". We also inquired about the perceived difficulties during discharge transition, factors that promote or impede recovery, and participants' needs (Table 2). The interview guide was suggested as a supplementary file.

Table 2

Interview topic guide
$<1$ or 2 days before discharge $>$
1) Describe your experience including readiness during discharge periods.
2) What are barriers you have perceived during discharge periods?
3) What are facilitators you have perceived during discharge periods?
4) What efforts do you make for healthy discharge transition?
5) Tell me information, education, support to want to provide.
6) Is there anything else you feel is important to mention?
< average 4 weeks after discharge>
1) Describe your experience including activities of daily living after discharge.
2) What are barriers you have perceived after discharge periods?
3) What are facilitators you have perceived after discharge periods?
4) Describe any change or difference after discharge periods.
5) What efforts do you make for adaptation of activities of daily living?
6) Tell me information, education, support to want to provide.
7) Is there anything else you feel is important to mention?

All the interviews were conducted by the first author who has attended qualitative research classes and related workshops and has experience in conducting qualitative research. A research assistant played an auxiliary role including recording during the interview process. The time required for each individual interview was approximately 30-40 min, and all interviews were audio-recorded with the participants' consent. The recorded interviews were only accessible to the interviewers, and the participants were informed that the recordings would be deleted immediately after the research. Interviews were conducted until data saturation was achieved. The researchers carefully listened to the participants and recorded meaningful statements as written notes during the interviews. Additionally, the researchers asked further questions if the participants' statements were ambiguous. Recorded material was transcribed verbatim by a research assistant trained in qualitative data analysis. Transcribed interview data and field notes were then analyzed.

\section{Data Analysis}

We employed the content analysis method (27) using NVivo 11 (QSR International, Melbourne, Australia). Meaningful data were extracted after reading the transcribed interviews and field notes several times and were coded through constant comparative analysis (28) to identify similarities and differences between data segments. The coded data were classified based on similarities and integrated into categories. The commonalities and natural changes in the data were identified and themes were derived as abstract entities from the categories. We repeated the process of reading and reviewing the original data again to clarify the relevance of the derived categories and key themes, which were refined, as necessary.

\section{Ethical Considerations}

This study complied with the principles laid down in the Declaration of Helsinki and was approved by the institutional review board (DHUMC-D-17024-PRO-02). Written consent was provided and obtained from all participants before study commencement. Confidentiality and privacy were guaranteed and each interview file was coded and anonymized.

\section{Rigor}

Page 5/13 
Trustworthiness of this study was established through prolonged engagement, triangulation of data sources, member checking, peer review, detailed description, and external auditing (29). To assure credibility, participants were interviewed twice, and an additional telephone interview was conducted when necessary. This allowed for a relationship to be built between the interviewer and the interviewee, and provided an opportunity to revise any misinformation. For data triangulation, we compared the interview transcripts with the field notes. Concerning member checking, an older adult's and a primary caregiver's views were taken on the study findings to ensure credibility. For peer review, the second author, who is experienced in qualitative research, repeatedly reviewed the results to ensure the quality and validity of the analysis at all stages. All authors discussed the findings until agreement was reached. To enhance transferability, we provided a detailed description of the setting, participants' characteristics, and the selection methods. To ensure dependability and confirmability, a professor of nursing with substantial experience in qualitative research examined the process, analyses, and findings. The Consolidated Criteria for Reporting Qualitative Research (COREQ) checklist was used to report the findings (30).

\section{Results}

The analysis yielded four main themes and ten categories. Each theme is illustrated using excerpts from the interviews.

\section{Theme 1: Challenge of discharge transition}

\section{1) Unprepared discharge}

Participants recognized their unstable state at the time of discharge and wanted to stay longer in the surgery hospital. Older adults (5 of 12) were forced to complete discharge from the surgery hospital despite their limited mobility. They believed that the recovery was delayed owing to the short hospitalization period after surgery. They expressed feeling upset at being discharged so soon.

I was discharged after a mere four and a half days; so, I felt [I received] unfair treatment. How [can you discharge] a person who has [had] a major operation so soon! That's sad (Interviewee 8).

\section{2) Transfer into other care settings}

Most older adults (10 of 12) moved to another care setting after discharge. Four participants faced difficulties in finding their own care setting without help and lacked pertinent information. Participants who moved to another care setting reported that they received care unrelated to the therapeutic instructions.

I said I could exercise, but they said, "no." When I asked why not, they told me that they would not allow me to do it because they were in a situation where they had to take responsibility about going wrong (Interviewee 6).

Some participants (four older adults) had to make alterations to their living environment because of mobility limitations.

My house is on the fourth floor; so, the thing to worry about now is whether I have to move to a house with an elevator (Interviewee 9).

\section{3) Eagerness for recovery}

Older adults (6 of 12) showed eagerness for recovery to cope with the challenge of discharge transition. They commented on nutritional management, rehabilitative exercise, walking, and adhering to physician's orders.

I have to walk using a walker now and turn around. I have to focus on this. I'm ready to make it fast. I have to exercise, eat well, and get better every day (Interviewer 12).

Two participants tried to understand and accept their diseases instead of denying them to improve their recovery process.

When I think that even young people fall and come to the hospital, I can also do that! Anyone can experience that! It is nice to have a new artificial joint inserted. After the surgery, I said to myself to get along with the artificial joint (Interviewee 2).

\section{Theme 2: Physical and psychological distress against recovery}

\section{1) Frail physical state}

Page 6/13 
Participants were in a frail physical state. Ten out of the twelve participants had multiple or severe underlying diseases including osteoporosis. They were using polypharmacy before the fracture owing to serious underlying diseases and had poor health status. Severe underlying diseases affected the patients' recovery process after hip fracture surgery, and they continued to be in a poor state post discharge.

I have diabetes, poor kidneys, higher blood pressure, and bad eyes. Diabetes has been around for 50 years. Only $30 \%$ of my kidneys remain (Interviewee 8 ).

They did not eat enough owing to a limited appetite, digestion, or diet due to the underlying disease.

Actually, I do not eat well. I say, "I do not want to eat" when my child asks me what I want to eat. Whenever I try to eat, I get tired easily. I'm going to ask for some medicine [to improve my] appetite (Interviewee 5).

\section{2) Psychological difficulties}

Some participants also experienced psychological difficulties. Frail physical state negatively affected their confidence in recovery. The worse the frail physical state, the more they fell into despair (6 of 12), which hindered their recovery. The following interviewee was informed that her walking should be limited for a month due to severe osteoporosis, and she showed frustration related to movement restriction.

I [was] unpleasantly surprised when I heard that I should not walk for a month or so. I knew I would walk if it is possible to walk little by little. Then, I wanted to go to the bathroom. I wanted to do it gradually. I took a leak and shat in my bed without moving. Oh! How waslchanged miserably like this? (Interviewee 4)

Other older adults (2 of 12$)$ perceived their current health condition as a punishment.

"Did I commit [a] sin?" People said repeatedly "it is not a sin."... Getting sick like this? I think I was punished (Interviewee 9).

\section{Theme 3: Dependent compliance}

\section{1) Absolute trust in healthcare providers}

Participants sought care at a tertiary hospital because the secondary hospitals were reluctant to perform surgery for patients who may have had underlying diseases, or they had had successful experiences-directly or indirectly-at tertiary hospitals. Most participants (10 of 12) expressed gratitude for undergoing surgery and were satisfied with the successful surgery.

What if I get broken and do not get surgery? I'm feeling good because I got surgery (Interviewee 12).

Older adults (6 of 12) showed strong trust in healthcare providers and noted their willingness to follow their therapeutic instructions.

All I can do is listen to the doctor's words and do it. There is only that (Interviewee 10).

\section{2) Indispensable support from the family}

All participants received supported from family members throughout the discharge transition. Some (2 of 12) had home-visit care services including support for physical and household activities in their homes. This help was also available to older adults. However, they could not continue their daily life without family support. Family members were primary caregivers who mainly helped activities of daily life and encouraged exercise and diet.

My son brought some meat for me, saying I should eat beef, beef, beef. I should eat because of my son. I'm about to [shed] a tear when I think of my son (Interviewee 5).

Older adults felt grateful for family support, while some older adults (3 of 12) also felt the burden of being cared for.

I'm sorry for my child. My child would not suffer that way if I died early. I'm worried about how much the hospital bill will be (Interviewee 8).

\section{3) Passive participation in care}


Most participants (8 of 12) received insufficient information about postoperative management owing to staff members' busy schedules or lack of interest. Some participants had difficulty in reading text-based discharge information.

Even if my son asked me to keep reading, I didn't read the text because it was difficult to read (Interviewee 9).

Nevertheless, they expressed a vague expectation that it would get better with time. Some medical staff explained the surgery process to the caregivers instead of the older adults.

I do not know well because my daughter is listening (Interviewer 7).

\section{Theme 4: Walking for things they took for granted}

\section{1) Hope of walking}

The participants ( 6 of 12) experienced their physical recovery, while others did not. Physical recovery indicated reduced pain, improved physical condition, and starting to move little by little.

I have a little more energy now than when I was discharged, and the hips hurt less. (Interviewee 11).

Most participants (9 of 12) described that being able to walk was their main wish during the discharge transition. Their expectations for walking ranged from using toilet independently or with walking aids, returning to their home to going to the farm according to the current status of the physical recovery.

Can I walk or not, getting hurt like this?Walking around on feet. Right, it would be better if I go to the bathroom with my feet (Interviewee 4).

The purpose of improving the walking ability was that participants could perform simple daily chores. Performing tasks they had earlier taken for granted was an essential part of daily life.

I just want to go home and cook for the poor youngest (Interviewee 1).

\section{2) Poor walking ability}

Despite the hope of walking, the walking ability of the participants was poor during the discharge transition period; some older adults (4 of 12) had mobile status indoors or outdoors using walking aids, while most (7 of 12) had immobile status indoors and outdoors. In addition to the frail physical state, there were some direct factors affecting walking ability. Severe pain hindered their recovery (7 of 12). Most older adults (9 of 12) were also afraid to move owing to fear the of falling and subsequent re-fracture, even though the pain significantly reduced.

There is nothing painful now. But I'm afraid to take a step.I can't take a step. I do not think I can get up (Interviewee 9).

\section{Discussion}

The purpose of this study was to clarify older adults' experience of discharge transition after hip fracture surgery. We identified four main themes-challenge of discharge transition, physical and psychological distress against recovery, dependent compliance, and walking for things they took for granted. This study is significant as it elucidates patients' distinct experiences, which inform future discharge transition programs.

First, challenge of discharge transition included three categories-unprepared discharge, transfer into other care settings, and eagerness for recovery. Consistent with a prior study (6), most older adults believed that their state was unstable and wanted to stay longer in the surgery hospital. Short hospitalization as a measure for cost-saving is currently the rule rather than the exception. Post discharge, most older adults had to enter other care settings. They experienced unconnected care in these other care facilities. Fragmented care makes it difficult for older adults' needs to be met (11).

Enhanced recovery programs in collaboration with healthcare professionals $(31,32)$, suitable and effective contents, and delivery of discharge teaching (33) are likely to helpful for promoting discharge readiness. A qualitative systematic review (26) also found that 
nurses played a significant role in coordinating between different health care settings, and post-discharge intervention was necessary for facilitating safe discharge transition. The current finding that some older adults struggled to recover functions such as exercising and eating was consistent with a previous study (19), which reported that older adults began to plan for their recovery immediately after hip fracture surgery because regaining independence was the most important issue in their daily life (19).

As a second theme, physical and psychological distress against recovery was indicated. Older adults were in a frail physical state. They already had underlying diseases including osteoporosis, and were malnourished owing to poor appetite, digestion, and limited diet due to underlying diseases, even though they recognized the importance of food intake (34). The frailty likely made their recovery more difficult. Osteoporosis, which most older adults with hip fracture have, is closely correlated with sarcopenia. Combining osteoporosis and sarcopenia may cause hip fracture $(35,36)$. Thus, interventions for sarcopenia as well as osteoporosis are essential for improving their frail physical state including exercise, nutritional support, and pharmacological treatments $(35,36)$.

Further, frail physical state caused psychological distress including loss of confidence and frustration. This was consistent with a previous study (37), which demonstrated that frailty was the most important prognostic factor for depression and anxiety in patients with hip fracture. Psychological distress plays a major role in interfering with treatment after hip fracture $(37,38)$. To overcome discharge transition instability, not only physical but also psychological support may prove helpful.

The third theme, dependent compliance includes absolute trust in healthcare providers, indispensable support from the family, and passive participation in care. The result regarding absolute trust in healthcare providers was similar to a previous study (19) in which older adults expressed their need to feel supported by healthcare providers. Older adults also depended entirely on family members, likely because of the family-centered culture (39) and limited social interaction (4), although some had home-visit care services during limited hours in their home.

Older adults were rather passive participants in their own care. They were either partly or not at all involved in the plan for treatment and recovery because managing their own illnesses was complex and challenging (11). In Eastern cultures, physicians often tend to explain care plans to the family rather than individual patients (39). In this study, older adults wanted to feel supported and at the same time found it difficult to participate in their own care. Feeling supported is likely to be achieved when nurses invite older adults for participation in their care $(11,39)$. Family involvement is also important in family-oriented cultures $(39)$. Thus, invitation for care participation by older adults as well as family involvement should be considered at discharge transitional care in family-centered cultures.

The final theme was walking for things they took for granted. The purpose of walking recovery was to perform simple daily chores. It is consistent with a previous study demonstrating that an essential requirement for daily functioning is recovering walking (40). Their expectation for walking was different according to their current ambulation state. The walking ability of older adults after hip fracture was affected by various factors: underlying diseases $(24,41,42)$, pre-fracture mobility $(41,42)$, age (42), and health status (43).

In addition to the physical and psychological distress mentioned above, severe pain and fear of falling acted as direct factors affecting walking ability in this study. Pain has been known as a major barrier for early recovery and should be treated as soon as possible (44). However, older adults with hip fracture are often not actively treated for pain during their hospitalization because of difficulty of assessment and concerns regarding drug complications (45). Tailored pain management is needed for safe and adequate pain relief $(44,45)$, considering that prolong pain was independently associated with catastrophic decline of walking recovery $(40)$.

Older adults also complain of fear of falling at onset of recovery. A previous study (16) reported that the fear of falling was the primary constraint in the poorest ADL group and instrumental ADL group. Older adults continued to complain of fear of falling even 3-6 months after hip fracture surgery (16), and after completion of a 4-6 week rehabilitation program (46). Considering that fear of falling is a factor that hinders walking ability from onset to long-term recovery, assessment and intervention to reduce fear of falling should be initiated as soon as possible after surgery.

In this study, most participants had immobile status indoors and outdoors during the discharge transition periods even though they were mobile before fracture. Most older adults have difficulty walking at discharge after hip fracture surgery (7) and their functioning does not return to the pre-fracture state even 1 year after surgery (12). Delayed partial weight-bearing after surgery has been reported to predict walking failure after hip fracture surgery (41). For effective physical rehabilitation including partial weight bearing at the earliest possible time, nursing needs to collaborate and coordinate with interdisciplinary personnel $(26,31)$. 


\section{Limitations}

This study had several limitations, First, the sample was recruited from a tertiary teaching hospital in Korea using convenience sampling; thus, our results may not be generalizable. Second, all older adults in our study were women. Although hip fracture caused by low-energy fall is four times more common in women than in men (7), it is necessary to examine whether gender differences exist in the experience of discharge transition after hip fracture surgery. Third, our sample size was small. Moreover, three participants declined to participate after discharge owing to an older adult's death and no outpatient visits. This variance may have affected the results. Lastly, our findings cannot be extended to countries with different healthcare systems and cultures.

\section{Conclusion}

In this study, experience of discharge transition by older adults after hip fracture helps nurses improve transitional care in acute settings. After hip surgery, older adults hoped to be able to walk for simple daily chores that they earlier took for granted. Considering the physical and psychological frailty of older adults undergoing hip surgery, systematic nursing interventions including collaboration and coordination with other healthcare professionals and settings are necessary during discharge transition after hip fracture surgery. Invitation for care participation by older adults as well as family involvement should be considered at discharge transitional care in South Korea. We propose a follow-up study to evaluate the experience of recovery of older adults by increasing post-discharge periods, considering the inevitability of long-term recovery after hip fracture surgery.

\section{Abbreviations}

ADL - Activities of Daily Living

\section{Declarations}

\section{Ethics Declarations}

- Ethics approval and consent to participate

This study was approved by the institutional review board (DHUMC-D-17024-PRO-02).

Written consent was obtained from all participants before study commencement.

- Consent for publication

Not applicable

- Availability of data and materials

Data is available upon reasonable request from the first and corresponding authors.

- Competing interests

The authors declare no potential conflicts of interest

- Funding

This study was supported by the National Research Foundation of Korea (NRF) funded by the Ministry of Science, ICT, and Future Planning (NRF-2017R1C1B5075973).

- Authors' contributions

Conception and design. YJ.

Data collection, analysis and interpretation of data. $\mathrm{YJ}, \mathrm{JH}$, and $\mathrm{SH}$.

Preparing, drafting, and editing. $\mathrm{YJ}, \mathrm{JH}$, and $\mathrm{SH}$.

Page $10 / 13$ 
All authors read and approved the final manuscript.

- Acknowledgements

We gratefully acknowledge the contribution of all the respondents who participated in the study.

- Declaration of Conflicting Interests

Author(s) declared no potential conflicts of interest with respect to the research, authorship, and/or publication of this article.

\section{References}

1. Kanis JA, Oden A, McCloskey EV, Johansson H, Wahl DA, Cooper C. A systematic review of hip fracture incidence and probability of fracture worldwide. Osteoporosis Int. 2012;23(9):2239-56. doi: 10.1007/s00198-012-1964-3

2. Cameron ID, Chen JS, March LM, Simpson JM, Cumming RG, Seibel MJ, et al. Hip fracture causes excess mortality owing to cardiovascular and infectious disease in institutionalized older people: A prospective 5-year study. J Bone Miner Res.

2010;25(4):866-72. doi:10.1359/jbmr.091029

3. Gao H, Liu Z, Xing D, Gong M. Which is the best alternative for displaced femoral neck fractures in the elderly?: A meta-analysis. Clin Orthop Relat Res. 2012 Jun;470(6):1782-91. doi: 10.1007/s11999-012-2250-6

4. Reay PA, Horner B, Duggan R. The patient's experience of early discharge following total hip replacement. Int $J$ Orthop Trauma Nurs. 2015;19(3):131-9. doi: 10.1016/j.ijotn.2015.02.003

5. Şendir M, Büyükyılmaz F, Muşovi D. Patients' Discharge information needs after total hip and knee arthroplasty: A quasiqualitative pilot study. Rehabil Nurs. 2013;38(5):264-71. doi: 10.1002/rnj.103

6. Brent L, Coffey A. Patient's perception of their readiness for discharge following hip fracture surgery. Int $\mathrm{J}$ Orthop Trauma Nurs. 2013;17(4):190-8. doi: 10.1016/j.ijotn.2013.01.001

7. Handoll HH, Sherrington C, Mak JC. Interventions for improving mobility after hip fracture surgery in adults. Cochrane Database Syst Rev. 2011 Mar 16(3):CD001704. doi: 10.1002/14651858.CD001704.pub4

8. Sjøveian AKH, Leegaard M. Hip and knee arthroplasty-patient's experiences of pain and rehabilitation after discharge from hospital. Int J Orthop Trauma Nurs. 2017;27:28-35. doi: 10.1016/j.ijotn.2017.07.001

9. Sveinsdóttir H, Skúladóttir H. Postoperative psychological distress in patients having total hip or knee replacements: An exploratory panel study. Orthop Nurs. 2012;31(5):302-11. doi: 10.1097/NOR.0b013e318266496f

10. Nahm E, Resnick B, Orwig D, Magaziner J, DeGrezia M. Exploration of informal caregiving following hip fracture. Geriatr Nurs. 2010;31(4):254-62. doi: 10.1016/j.gerinurse.2010.01.003

11. Rustad EC, Furnes B, Cronfalk BS, Dysvik E. Older patients' experiences during care transition. Patient Prefer Adherence. 2016 May 12;10:769-79. doi: 10.2147/PPA.S97570

12. Córcoles-Jiménez MP, Villada-Munera A, del Egido-Fernández MÁ, Candel-Parra E, Moreno-Moreno M, Jiménez-Sánchez MD, et al. Recovery of activities of daily living among older people one year after hip fracture. Clin Nurs Res. 2015 Dec;24(6):604-23. doi: $10.1177 / 1054773815573261$

13. Meleis Al, Sawyer LM, Im EO, Hilfinger Messias DK, Schumacher K. Experiencing transitions: An emerging middle-range theory. Adv Nurs Sci. 2000 Sep;23(1):12-28. doi: 10.1097/00012272-200009000-00006

14. Altfeld SJ, Shier GE, Rooney M, Johnson TJ, Golden RL, Karavolos K, et al. Effects of an enhanced discharge planning intervention for hospitalized older adults: A randomized trial. Gerontologist. 2013;53(3):430-40. doi: 10.1093/geront/gns109

15. Wells M, Wade M. Physical performance measures: An important component of the comprehensive geriatric assessment. Nurse Pract. 2013;38(6):48-53. doi: 10.1097/01.NPR.0000429895.41373.7e

16. Ko Y, Lee J, Kim SY, Baek SH. Identification of factors related to functional decline of Korean older adults after hip fracture surgery: A cross-sectional study. Res Gerontol Nurs. 2019;12(6):312-20. doi: 10.3928/19404921-20190702-03

17. Cobley CS, Fisher RJ, Chouliara N, Kerr M, Walker MF. A qualitative study exploring patients' and carers' experiences of Early Supported Discharge services after stroke. Clin Rehabil. 2013;27(8):750-7. doi: 10.1177/0269215512474030

18. Schiller C, Franke T, Belle J, Sims-Gould J, Sale J, Ashe MC. Words of wisdom - patient perspectives to guide recovery for older adults after hip fracture: a qualitative study. Patient Prefer Adherence. 2015 Jan 12;9:57-64. doi: 10.2147/PPA.S75657

Page $11 / 13$ 
19. Segevall C, Söderberg S, Randström KB. The journey toward taking the day for granted again: The experiences of rural older people's recovery from hip fracture surgery. Orthop Nurs. 2019;38(6):359-66. doi: 10.1097/NOR.0000000000000608

20. Sims-Gould J, Stott-Eveneshen S, Fleig L, McAllister M, Ashe MC. Patient perspectives on engagement in recovery after hip fracture: a qualitative study. J Aging Res. 2017 Mar 20;2017. doi: 10.1155/2017/2171865

21. Yoo J, Lee J, Kim S, Kim B, Choi H, Song D, et al. Length of hospital stay after hip fracture surgery and 1-year mortality. Osteoporosis Int. 2019;30(1):145-53. doi: 10.1007/s00198-018-4747-7

22. Leland NE, Gozalo P, Christian TJ, Bynum J, Mor V, Wetle TF, et al. An examination of the first 30 days after patients are discharged to the community from hip fracture postacute care. Med Care. 2015 Oct;53(10):879-87. doi: 10.1097\%2FMLR.0000000000000419

23. Shyu $\mathrm{YL}$, Chen $\mathrm{M}$, Wu $\mathrm{C}$, Cheng $\mathrm{H}$. Family caregivers' needs predict functional recovery of older care recipients after hip fracture. J Adv Nurs. 2010;66(11):2450-9. doi: 10.1111/j.1365-2648.2010.05418.x

24. Kim S, Sok SR. Relationships among the perceived health status, family support and life satisfaction of older Korean adults. Int $J$ Nurs Pract. 2012;18(4):325-31. doi: 10.1111/j.1440-172X.2012.02050.x

25. Statistics Korea. National health insurance statistics. 2017. http://kosis.kr/statHtml/statHtml.do? orgld=350\&tblld=TX_35001_A061\&vw_cd=\&list_id=\&scrld=\&seqNo=\&lang_mode=ko\&obj_var_id=\&itm_id=\&conn_path=K1\&path=. Accessed 21 Sept 2019.

26. Puls SE, Guerrero KS, Andrew DA. Facilitating safe patient transition of care: A qualitative systematic review. J Nurs Educ Pract. 2014 Jun 1;4(6):37. doi: 10.5430/jnep.v4n6p37

27. Polit DF, Beck CT. Nursing research: Generating and assessing evidence for nursing practice. USA: Lippincott Williams \& Wilkins; 2012.

28. Thorne S. Data analysis in qualitative research. Evid Based Nurs. 2000;3(3):68-70. doi: 10.1136/ebn.3.3.68

29. Lincoln YS, Guba EG. Establishing trustworthiness. Naturalistic Inquiry. 1985;289(331):289-327.

30. Tong A, Sainsbury P, Craig J. Consolidated criteria for reporting qualitative research (COREQ): A 32-item checklist for interviews and focus groups. Int J Qual Health Care. 2007;19(6):349-57. doi: 10.1093/intqhc/mzm042

31. Dusek B, Pearce N, Harripaul A, Lloyd M. Care transitions: A systematic review of best practices. J Nurs Care Qual. 2015;30(3):233-9. doi: 10.1097/NCQ.0000000000000097

32. Sibbern T, Bull Sellevold V, Steindal SA, Dale C, Watt-Watson J, Dihle A. Patients' experiences of enhanced recovery after surgery: A systematic review of qualitative studies. J Clin Nurs. 2017;26(9-10):1172-88. doi: 10.1111/jocn.13456

33. Nurhayati N, Songwathana P, Vachprasit R. Surgical patients' experiences of readiness for hospital discharge and perceived quality of discharge teaching in acute care hospitals. J Clin Nurs. 2019;28(9-10):1728-36. doi: 10.1111/jocn.14764

34. Li H, Cheng H, Liang J, Wu C, Shyu YL. Functional recovery of older people with hip fracture: Does malnutrition make a difference? J Adv Nurs. 2013;69(8):1691-703. doi: 10.1111/jan.12027

35. Oliveira A, Vaz C. The role of sarcopenia in the risk of osteoporotic hip fracture. Clin Rheumatol. 2015;34(10):1673-80. doi: 10.1007/s10067-015-2943-9

36. Wakabayashi H, Sakuma K. Rehabilitation nutrition for sarcopenia with disability: A combination of both rehabilitation and nutrition care management. J Cachexia Sarcopenia Muscle. 2014;5(4):269-77. doi: 10.1007/s13539-014-0162-x

37. van de Ree C, de Munter L, Biesbroeck B, Kruithof N, Gosens T, de Jongh M. The prevalence and prognostic factors of psychological distress in older patients with a hip fracture: A longitudinal cohort study. Injury. 2020. doi: 10.1016/j.injury.2020.07.049

38. Vincent HK, Horodyski M, Vincent KR, Brisbane ST, Sadasivan KK. Psychological distress after orthopedic trauma: Prevalence in patients and implications for rehabilitation. PM\&R. 2015;7(9):978-89. doi: 10.1016/j.pmrj.2015.03.007

39. Lin M, Pang MS, Chen C. Family as a whole: Elective surgery patients' perception of the meaning of family involvement in decision making. J Clin Nurs. 2013;22(1-2):271-8. doi: 10.1111/j.1365-2702.2012.04194.x

40. Salpakoski A, Törmäkangas T, Edgren J, Sihvonen S, Pekkonen M, Heinonen A, et al. Walking recovery after a hip fracture: a prospective follow-up study among community-dwelling over 60-year old men and women. BioMed Res Int. 2014 Jan 1;2014. doi: $10.1155 / 2014 / 289549$ 
41. Kondo A, Hagino H, Zierler BK. Determinants of ambulatory ability after hip fracture surgery in Japan and the USA. Nurs Health Sci. 2010;12(3):336-44. doi: 10.1111/j.1442-2018.2010.00535.x

42. Pioli G, Lauretani F, Pellicciotti F, Pignedoli P, Bendini C, Davoli M, et al. Modifiable and non-modifiable risk factors affecting walking recovery after hip fracture. Osteoporosis Int. 2016;27(6):2009-16. doi: 10.1007/s00198-016-3485-y

43. Kristensen MT. Factors affecting functional prognosis of patients with hip fracture. Eur J Phys Rehabil Med. 2011 Jun;47(2):25764.

44. Tosounidis TH, Sheikh H, Stone MH, Giannoudis PV. Pain relief management following proximal femoral fractures: Options, issues and controversies. Injury. 2015;46:S52-8. https://doi.org/10.1016/j.injury.2015.08.014

45. Hung WW, Egol KA, Zuckerman JD, Siu AL. Hip fracture management: Tailoring care for the older patient. JAMA. 2012;307(20):2185-94. doi:10.1001/jama.2012.4842

46. Visschedijk JH, Caljouw MA, Bakkers E, van Balen R, Achterberg WP. Longitudinal follow-up study on fear of falling during and after rehabilitation in skilled nursing facilities. BMC Geriatr. 2015;15(1):161. https://doi.org/10.1186/s12877-015-0158-1

\section{Supplementary Files}

This is a list of supplementary files associated with this preprint. Click to download.

- Casereportform.docx 